\title{
Italian Validation of Family Adaptability and Cohesion Scale (FACES IV) Short Version for Adolescents: SAD_FACES
}

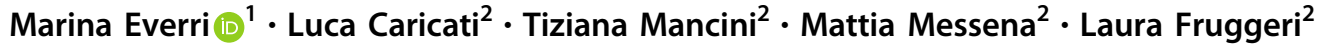

Received: 21 May 2019 / Revised: 17 June 2020 / Accepted: 22 June 2020 / Published online: 24 July 2020

(c) The Author(s) 2020

\begin{abstract}
This study validated the Italian short version of FACES-IV (Family Adaptability and Cohesion Scale) for adolescents, namely SAD_FACES. The scale assessed adolescents' perceptions of their families' adaptive and maladaptive functioning along the six dimensions of cohesion, flexibility, disengagement, enmeshment, rigidity and chaos as defined by the Circumplex Model of Marital and Family Systems. SAD_FACES was administrated to a sample of secondary school students (Age = 14-16 years; $N=446$ ). The Family Communication Scale (FCS), measuring positive communication skills used in the family system, was also administered to assess external validity. ESEM was performed and evaluation of model fit was based on values of the comparative fit index (CFI), the Tucker-Lewis Index (TLI), and the root mean square error of approximation (RMSEA). The scores that were computed as the sum of the intended items and latent traits were both considered for each dimension. Results showed that SAD_FACES (24 items) has the same structure and internal consistency of the Italian version of FACES IV validated with adolescent samples. Implications for the validity and usage of a short scale for the assessment of adolescents' perception of family functioning and their wellbeing are discussed. Future research should validate SAD_FACES with different age cohorts of adolescents and belonging to different cultural contexts as well as consider clinical samples of adolescents. The agility of SAD_FACES could facilitate investigations with clinical samples of adolescents. Future research is needed in this area.
\end{abstract}

Keywords FACES IV short form • Adolescents · FACES IV cross-cultural validation · Family functioning $\cdot$ Italy

\section{Highlights}

- FACES IV short version was cross-culturally validated in a sample of Italian adolescents.

- SAD_FACES assessed adolescents' perception of family functioning on a 24-items scale.

- SAD_FACES cross-cultural validation had the same structure and internal consistency of FACES IV.

- SAD_FACES could complement instruments with multiple scales as well as adolescents' clinical assessments.

The last version of the Family Adaptability and Cohesion Scale (FACES IV) was developed within the framework of the Circumplex Model of Marital and Family Systems with the aim to assess family adaptive and maladaptive functioning through a multi-dimensional approach (Olson 2011; Olson and Gorall 2006; Olson et al. 1979, 1989). The

Marina Everri

marina.everri@ucd.ie

1 School of Medicine, University College Dublin, Stillorgan Road, Belfield, Dublin 4, Ireland

2 University of Parma, Parma, Italy
Circumplex Model of Marital and Family Systems (Olson et al. 1989, 1979; Olson and Gorall 2003) has paved the way for the study of family functioning, namely the complex relational patterns that regulate everyday interactions among family members (Minuchin 1974). Cohesion and flexibility are the central dimensions of the model, plus communication as a facilitating dimension, which encompasses positive communication skills used in the family system (Barnes and Olson 1985).

Cohesion refers to the emotional closeness among family members; flexibility concerns the style of leadership and its expression in terms of roles, relationship rules and negotiations. Both cohesion and flexibility develop along a continuum with intermediate and extreme (low and high) 
levels. A low level of cohesion has been defined as disengagement, namely absent emotional bond; while a high level of cohesion has been defined as enmeshment, namely excessive emotional bond. A low level of flexibility has been defined as rigidity, namely strict family power, leadership, and rules; while a high level of flexibility has been defined as chaos, namely lax family power, leadership, and rules. Intermediate levels of cohesion and flexibility have been defined as balanced/moderate emotional closeness and balanced/moderate leadership.

FACES IV subscales were developed to tap the full continuum (high, intermediate, low levels) of cohesion and flexibility from the Circumplex Model. Six scales were developed, for a total of 42 items, with two balanced scales tapping intermediate levels of cohesion and flexibility and four unbalanced scales designed to tap, respectively low and high levels of cohesion (disengagement and enmeshment) and flexibility (chaos and rigidity). An additional scale on family communication assesses families' communication skills (Barnes and Olson 1985). Families showing intermediate/moderate levels of cohesion and flexibility were found to be well-functioning families (balanced families). Differently, families having either high or low levels of cohesion and flexibility, assessed through the unbalanced scales of FACES IV, have been found to be dysfunctional families (unbalanced families) (Everri et al. 2016b; Loriedo et al. 2013; Olson and Gorall 2006; Rivero et al. 2010). Additionally, research showed that communication is positively correlated with moderate cohesion and flexibility and negatively correlated with disengagement, enmeshment, rigidity, and chaos (Olson 2011).

In its initial version (Olson et al. 1985), FACES IV was validated and applied in samples of adults and young adults only (older than 18 years) to measure normative family functioning. However, its applicability in clinical contexts revealed to be useful for clients' initial assessment and integration with other diagnostic instruments (Loriedo et al. 2013; Olson 2011). A more recent strand of research has validated FACES IV with samples of adolescents (Baiocco et al. 2013; Everri et al. 2015, 2016a, 2016b). These studies demonstrated that FACES IV can provide further insights to the understanding of parent-child relationship as well as to the measurement of adolescents' wellbeing through the identification of risk factors during developmental transitions, such as adolescence (Everri et al. 2014; Larson et al. 1996). In fact, family functioning has been found to be connected with different aspects of adolescents' physical and psychological wellbeing as well as with school attainment (e.g., Berge et al. 2013; Shek 1997; Suldo and Huebner 2004). Consistently, balanced families can be considered as adaptive contexts for adolescents' positive development. However, when non-adult samples are concerned, additional aspects need to be taken into consideration.
In samples of adolescents, rigidity (strict rules and strong family leadership) can also be considered as an adaptive dimension, and its value depends on its association with other positive/negative dimensions of family functioning (Everri et al. 2016b). Younger adolescents tend to score higher than older adolescents on the unbalanced scales of enmeshment and rigidity, and females tend to have higher mean scores on the balanced scales of cohesion and flexibility than males (Baiocco et al. 2013). In addition, as pointed out by previous studies on short scales validation (e.g., Gosling et al. 2003; Postmes et al. 2013; Ziegler et al. 2014), the length of FACES IV (six scales and 42 items) can make it difficult to use this instrument with other questionnaires, especially when samples of children and adolescents are concerned.

Building upon research that have validated FACES IV in samples of adolescents (e.g., Baiocco et al. 2013; Everri et al. 2015, 2016a, 2016b; Henry et al. 2008), this study intended to provide a shorter and more agile version of the instrument to assess adolescents' perceptions of their family functioning. In so doing, we aimed to provide a tool for research with samples of adolescents as well as to further advance the literature on FACES IV validation in different age groups. Therefore, this study proposed and validated a short version of the FACES for adolescents, namely SAD_FACES.

Following the validity approach (i.e, Grimm and Widaman 2012), we were concerned with both internal and external validity of the scale. For internal validity, we were primarily interested in the analysis of the dimensionality and reliability of the scale (given that items have been largely used in past research, we considered content validity as already established). Thus, we analysed the factor structure of the items and their reliability. For external validity, we focused on concurrent validity by investigating correlations of FACES dimensions with family communication. We expected that family communication would be positively correlated with balanced dimensions (intermediate cohesion and flexibility) and negatively correlated with unbalanced dimensions (disengagement, enmeshment, rigidity, and chaos). With explorative purposes, we investigated gender differences in mean scores of FACES IV dimensions.

\section{Method}

\section{Participants}

The study sample consisted of 453 adolescents. Seven participants were not included because their answers to all items of the questionnaire or more than $80 \%$ of them were missing. The final sample comprised 446 students (age: $\mathrm{M}=14.37 ; \mathrm{SD}=0.75$; gender: $105,23.5 \%$ males; 339 , 
$76 \%$ female, 2 missing) attending the first 2 years of high school in a region of Northern Italy.

The majority of adolescents were born in Italy (404, $90.6 \%)$, lived in two married parent households $(344,78 \%)$ and had siblings $(342,76.7 \%)$. The adolescents' families belonged to the upper-middle class, their parents had a junior high school diploma (mother: $16.4 \%$, father: $27.9 \%$ ), a high school diploma (mother: 56.9\%; father: 50.6\%), a master or postgraduate degree (mother: $26.6 \%, 14$ missing; father $21.2 \%, 27$ missing).

\section{Procedure}

Questionnaires were administered in the classrooms over 1 hour in the presence of the teacher in charge and the researcher. Written consents for adolescents' participation in the study were obtained by parents via a signed letter before starting the data collection. Participation was voluntary and anonymous, and participants were encouraged to answer individually and as truthfully as possible.

\section{Measures}

\section{Item Selection}

In order to select items to be retained in the short version of the FACES IV (SAD_FACES), we used data from 277 adolescents to whom the Italian version (Baiocco et al. 2013) of the original FACES IV (Olson 2011) was administered for an unrelated research project. FACES IV contains 42 items assessed on a 5-point Likert scale that measures six dimensions: Two balanced subscales assess adaptive family functioning (i.e., intermediate levels of cohesion and flexibility), and four unbalanced subscales assess the highest and lowest extremes of the cohesion and flexibility continuum (respectively, enmeshment and disengagement, chaos and rigidity). As a reminder, disengagement refers to very low levels of cohesion and corresponds to the lowest extreme of the cohesion continuum; while enmeshment refers to very high levels of cohesion and corresponds to the highest extreme. Both represents maladaptive forms of cohesion and are considered unbalanced dimensions. Balanced cohesion, namely the adaptive form of cohesion, corresponds to an intermediate level between disengagement and enmeshment. The same principle applies for the dimension of flexibility. Thus, the dimensions of cohesion and flexibility do not represent simply the sum or the differences between different factors; rather, they represent the balanced score adjusted for the differences between unbalanced factors. Internal consistency in this study was similar to internal consistency of the Italian validation study (see Baiocco et al. 2013): balanced cohesion $(\alpha=0.75)$, balanced flexibility $(\alpha=0.70)$, enmeshment ( $\alpha=0.57)$, disengagement $(\alpha=0.77)$, chaos $(\alpha=0.57)$, and rigidity $(\alpha=0.73)$.

We aimed to retain four items for each FACES dimension. Given that FACES has been widely used and proved to be valid and reliable, we assumed that all items equally contributed to content and face-value validities of the scale. Thus, we selected items based on their psychometric properties. We used Rasch analysis in order to detect items that better measured the intended latent trait, given that Rasch analysis aims to "maximize the homogeneity of the trait and to allow greater reduction of redundancy at no sacrifice of measurement information by decreasing items and/or scoring levels to yield a more valid and simple measure" (Granger 2007, p. 1122). More specifically, we retained the four items that, for each dimension, contributed more to the measurement of the dimension having the better index of infit and outfit-two diagnostic indexes assessing the extent to which items are near or far from expected value - and being well suited with the mono-dimensionality of each dimension.

\section{Questionnaire}

\section{Socio-demographic data}

This section comprised questions aimed at collecting information about: adolescents' age, gender, household composition (number of family components), family structure (e.g., cohabiting/married parents, separated parents, step-parents), and parents' educational qualification and profession.

\section{Family functioning}

Adolescents completed the short version of Family Adaptability and Cohesion Evaluation Scale for adolescents (SAD_FACES). SAD_FACES contained 24 items that assess the six dimensions of the Olson's model on a 5-point Likert scale $(1=$ not at all; $5=$ definitely).

\section{Family communication}

To assess the concurrent validity of SAD_FACES, participants were also asked to complete the Family Communication Scale (FCS), which Barnes and Olson (1985) considered as a facilitating dimension for a positive family functioning. The FCS consists of 10 items measured on a five-point Likert scale where 1 indicates 'strongly disagree' and 5 'strongly agree' (Olson and Gorall 2006). We used the Italian version adapted by Baiocco and colleagues (Baiocco et al. 2013). Internal consistency was very good (Cronbach's $\alpha=0.90$ ). 


\section{Data Analytic Plan}

First, we checked for missing values on FACES and family communication items. Only $99(0.65 \%)$ missing values appeared on the database. Albeit some items had more missing values than other items, visual inspection suggested that no missing patterns were detectable. We tested for possible correlation of missing data with sex and age of participants and, accordingly, we found differences neither between genders $\left(\chi^{2}(1)=0.05, p=0.824\right)$ nor with respect to age (logistic regression $b=-0.07, \mathrm{SE}=0.17, Z=-0.39$, $p=0.696$ ). Thus, we assumed that missing values were at random and multiply imputed them with predictive mean matching procedure that is suitable also for categorical and non-normal distributed data. Multiple imputation was performed with mice package (van Buuren and GroothuisOudshoorn 2011) and R software (R Core Team 2019).

To assess the dimensionality aspect of internal validity, the expected six-dimension structure was evaluated with exploratory structural equation modelling (ESEM; Asparouhov and Muthén 2009; Marsh et al. 2014). ESEM was performed using the weighted least square mean- and variance-adjusted (WLSMV) estimator that allows taking in account the ordinal nature of items and provides robust parameter estimates and standard errors. In the tested model, items loaded on their intended factors, whereas cross-loadings were estimated with oblique target rotation procedure (e.g., Tóth-Király et al. 2017). According to Comrey and Lee (2013), we considered factor loadings of $0.71,0.63,0.55,0.45$ and 0.32 as excellent, very good, good, fair and poor respectively. Evaluation of model fit was based on values of the comparative fit index (CFI), the Tucker-Lewis Index (TLI), and the root mean square error of approximation (RMSEA) considering both adequate and good thresholds (e.g., Marsh 2007; Perry et al. 2015). Specifically, values greater than 0.90 and 0.95 were considered respectively adequate and excellent for both CFI and TLI, while values smaller than 0.08 and 0.06 indicated adequate and excellent RMSEA. We reported also chisquared test, although its value can be inflated with large sample size, and the chi-squared/degree of freedom ratio that should be lower than 3. Structural equation modelling was performed with Mplus 8.1 (Muthén and Muthén 1998-2017).

Again considering internal validity, reliability of dimensions was assessed with Cronbach's alpha and zeroorder correlations among dimensions were analyzed using Pearson's r. Moreover, for each dimension, we considered both the scores that were computed as the sum of the intended items and latent trait. Furthermore, following Olson et al.'s (2006) procedure, the sum scores were transformed in percentiles and then used to compute the score of both cohesion and flexibility dimensions. More precisely, cohesion dimension scores were computed as balanced cohesion $+($ enmeshment-disengagement $) \times 0.25$, while flexibility dimension scores were computed as balanced flexibility + (chaos-rigidity) $\times 0.25$. These values supply indexes of perceived general cohesive and flexible function of one's own family. These two scores then represent the scores of balanced dimensions adjusted for unbalanced (i.e., extreme) dimensions.

In order to consider external validity, we analysed zeroorder correlations between dimensions of the FACES and family communication with Person's product-moment correlation coefficient. Lastly, we explored gender differences on the FACES dimensions with multivariate analysis of variance. This was done with the exploratory purpose to observe whether gender would affect scores of FACES.

\section{Power and sample size concerns}

Rule of thumb suggests that an adequate sample size require more than 200 participants and/or a ratio of $\mathrm{N}$ to the number of variables in the model $>10$. In our case, both conditions were satisfied ( $\mathrm{N}=446$ and 24 items). However, given that there is not an agreement on sample size calculation for explorative and confirmatory factor analysis (e.g., Myers et al. 2011), we ran a Monte Carlo simulation using estimates from previous analysis as starting values. It is worth noting that Monte Carlo simulation for rotated solution risks tend to be incompatible with the notion of simplicity (Muthen and Muthen 1998-2017, see also Asparouhov and Muthén 2009). Thus, we ran Monte Carlo simulation (1000 replications) on a model in which latent dimensions were measured by the four intended items only. Results indicated that the smallest estimate that had a probability to be detected with $80 \%$ of power was 0.20 . This seems to confirm that our sample size is large enough to supply adequate power.

\section{Results}

\section{Internal Validity: Testing the Factor Structure of the FACES}

ESEM results showed that the expected 6-dimension structure fitted the data well $\left(\chi^{2}(147)=192.75, \chi^{2} / d f=\right.$ 1.31, $p=0.007, \mathrm{CFI}=0.991, \mathrm{TLI}=0.984, \mathrm{RMSEA}=$ $0.026, p=0.999,90 \%$ CI[0.015-0.036]). Table 1 shows standardized estimates for all items on all dimensions. As one can see, a clear six-factor structure emerged although some unexpected loadings appeared. In particular, one balanced cohesion item had poor fit on the balanced cohesion factor and a fair loading on balanced flexibility factor. Similarly, also one item from enmeshment dimension had 
Table 1 ESEM standardized estimates for all items on all dimensions of FACES IV

\begin{tabular}{|c|c|c|c|c|c|c|}
\hline & Cohesion & Flexibility & Disengagement & Enmeshment & Rigidity & Chaos \\
\hline $\mathrm{F} 1 \mathrm{CO}$ & $0.51 * *$ & $0.16 * *$ & $-0.18 * *$ & -0.03 & $0.13 * *$ & 0.06 \\
\hline F7CO & $0.50 * *$ & $0.12 *$ & $-0.25^{* *}$ & $0.18 * *$ & 0.01 & -0.08 \\
\hline $\mathrm{F} 20 \mathrm{CO}$ & $0.41 * *$ & $0.13 * *$ & $-0.19 * *$ & $0.23 * *$ & $0.09 *$ & $-0.14 * *$ \\
\hline $\mathrm{F} 13 \mathrm{CO}$ & $0.20 * *$ & $0.46^{* *}$ & $-0.14 *$ & $0.16^{* *}$ & -0.04 & $-0.14 *$ \\
\hline F24FL & -0.06 & $0.67 * *$ & $-0.16^{* *}$ & 0.05 & $0.11 * *$ & 0.06 \\
\hline $\mathrm{F} 22 \mathrm{FL}$ & 0.11 & $0.67 * *$ & $0.16^{* *}$ & 0.05 & 0.04 & -0.08 \\
\hline F8FL & $0.14^{*}$ & $0.54 * *$ & $-0.16 * *$ & -0.02 & -0.06 & 0.09 \\
\hline $\mathrm{F} 2 \mathrm{FL}$ & $0.36^{* *}$ & $0.32 * *$ & -0.01 & 0.02 & $0.10^{*}$ & -0.07 \\
\hline F14DI & -0.04 & 0.11 & $0.86^{* *}$ & 0.00 & 0.01 & 0.03 \\
\hline F3DI & $-0.19 * *$ & -0.07 & $0.63 * *$ & 0.06 & -0.02 & -0.02 \\
\hline F9DI & $-0.25 * *$ & 0.00 & $0.57 * *$ & 0.06 & 0.03 & $0.21 * *$ \\
\hline F18DI & 0.12 & $-0.25 * *$ & $0.45 * *$ & -0.01 & 0.03 & $0.18 *$ \\
\hline $\mathrm{F} 21 \mathrm{EN}$ & 0.06 & -0.03 & -0.04 & $0.72 * *$ & -0.01 & -0.10 \\
\hline F15EN & $-0.14^{*}$ & -0.01 & 0.08 & $0.63 * *$ & -0.03 & $0.24 * *$ \\
\hline F4EN & 0.08 & -0.04 & $0.14 *$ & $0.53 * *$ & -0.01 & -0.12 \\
\hline F10EN & $0.39 * *$ & -0.01 & -0.05 & $0.21 * *$ & $0.13 * *$ & $0.16^{*}$ \\
\hline F5RI & $-0.18 * *$ & $-0.15^{* *}$ & $-0.14 * *$ & -0.01 & $0.96 * *$ & 0.05 \\
\hline F11RI & 0.02 & -0.08 & -0.01 & 0.02 & $0.75 * *$ & $0.16^{* *}$ \\
\hline F23RI & -0.01 & $0.26^{* *}$ & 0.10 & -0.09 & $0.71 * *$ & $-0.20^{* *}$ \\
\hline F16RI & $0.19 * *$ & 0.04 & $0.22 * *$ & 0.08 & $0.60 * *$ & -0.08 \\
\hline $\mathrm{F} 6 \mathrm{CH}$ & -0.09 & 0.06 & -0.01 & 0.01 & 0.04 & $0.66^{* *}$ \\
\hline $\mathrm{F} 12 \mathrm{CH}$ & -0.12 & 0.07 & 0.05 & $0.13 *$ & -0.04 & $0.54 * *$ \\
\hline $\mathrm{F} 17 \mathrm{CH}$ & $0.18 * *$ & -0.06 & $0.22 * *$ & -0.04 & -0.05 & $0.58 * *$ \\
\hline $\mathrm{F} 19 \mathrm{CH}$ & -0.02 & 0.05 & 0.07 & -0.01 & -0.05 & $0.44 * *$ \\
\hline
\end{tabular}

$* p<0.05 ; * * p<0.01, N=446$ poor loadings. Additionally, one item of the balanced flexibility factor had fair loadings on both balanced flexibility and balanced cohesion factors. Albeit these unexpected loadings, we believe that the factor structure of the scale can be substantially confirmed.

\section{Internal Validity: Analysis of the Dimensions of the FACES}

For each latent dimension, score was computed as both sum of intended items and ESEM latent trait scores. Table 2 shows zero-order correlations, reliability indexes and descriptive statistics of the six dimensions of FACES (both sum and factor scores) and family communication. As one can see, scores that were computed as the sum of items were strongly correlated with latent trait scores ( $r$ s ranged from 0.89 to 0.98 ) suggesting that the four intended items supply most of the information that is supplied by all items on each dimension. Reliability of dimensions (when computed as sum of items) was satisfactory except for enmeshment and chaos. Reliabilities were not improved substantially by the exclusion of any item. In addition, correlations between dimensions were similar for sum and factor scores.
Additionally, the majority of correlations among the considered dimensions were in the expected direction. Cohesion and flexibility (balanced dimensions) were strongly and positively correlated, confirming concordance of functioning of balanced families. Cohesion was also negatively correlated with disengagement (lowest extreme of the cohesion continuum) and positively correlated with enmeshment (highest extreme of the cohesion continuum). The extreme unbalanced cohesion scales of disengagement and enmeshment were not significantly correlated. Similarly, balanced flexibility was negatively correlated with chaos (highest extreme of the flexibility continuum) and positively correlated with rigidity (lowest extreme of the flexibility continuum). Also in this case, unbalanced flexibility scales were not significantly correlated, confirming the independence and value of unbalanced extremes of both cohesion and flexibility (Olson 2011).

\section{External Validity: Communication and Gender Differences}

As expected, communication was positively correlated with balanced cohesion and balanced flexibility and negatively correlated with chaos and disengagement. Unexpectedly, 


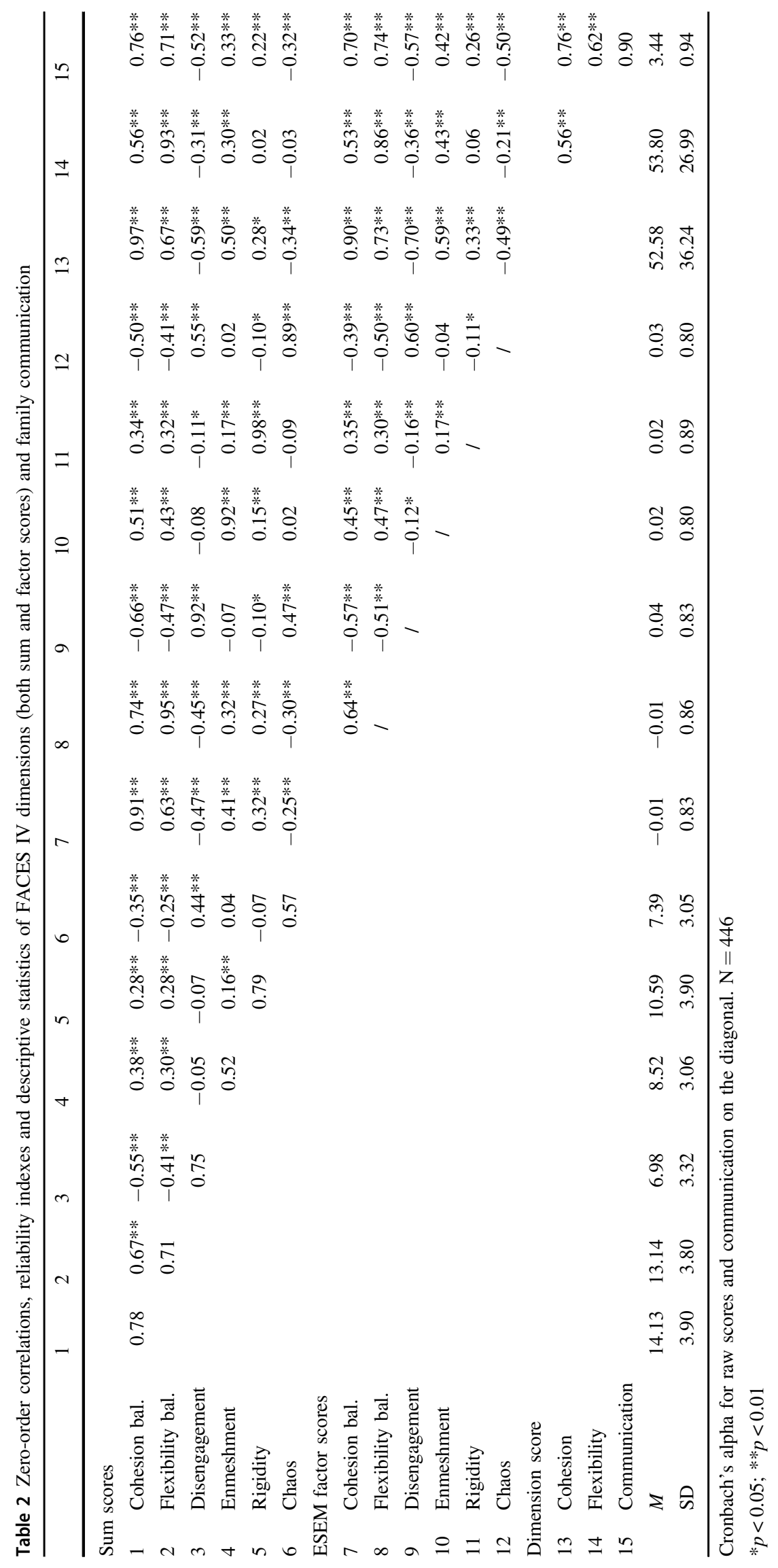


communication was positively correlated with the unbalanced scales of enmeshment and rigidity. It is worth noting, however, that communication was positively and significantly correlated with cohesion and flexibility dimensions (that are adjusted for unbalanced scores) and this suggests that, regardless of the degree of unbalance, moderately cohesive and flexible families are characterized by positive communication.

Lastly, we tested whether boys and girls differed in their scores on the six dimensions of FACES IV with multivariate analysis of variance. No significant multivariate effect appeared, Wilks' $\lambda=0.976, F(6,437)=1.782, p=$ 0.101 . Univariate results indicated that boys and girls differed only on the rigidity dimension $(F(1,442)=4.61, p=$ $\left.0.032, \eta^{2}=0.01\right)$ with boys scoring higher $(M=2.83$, $\mathrm{SD}=0.86)$ than girls $(M=2.56, \quad \mathrm{SD}=1.01)$. Similar results were obtained when considering factor scores.

\section{Discussion and Conclusions}

The present study intended to validate the Italian short version of FACES IV, an instrument used to assess family adaptive and maladaptive functioning. For this purpose, a sample of middle adolescents (14-16 years) was considered building upon recent studies that demonstrated the usefulness of FACES IV to assess youngsters' perceptions of their families' adaptive and maladaptive functioning (e.g., Everri et al. 2016a; Baiocco et al. 2013).

The analysis showed that the factor structure and dimensions reliability of the SAD_FACES confirmed FACES IV structure validated with samples of Italian adolescents. In addition, the majority of correlations among the considered dimensions were similar to those found in previous studies with adolescent samples using FACES IV. Enmeshment and rigidity, instead, showed positive correlations with balanced cohesion and flexibility. However, the positive correlation between balanced cohesion and enmeshment is consistent with previous studies that found both significant (Loriedo et al. 2013) and non significant but positive correlations (e.g., Baiocco et al. 2013; Graça Pereira and Teixeira 2013) between these two dimensions. As for rigidity, previous studies found that according to adolescents rigidity can be considered as a positive dimension of family functioning (Everri et al. 2015, 2016a, 2016b). This was confirmed by the observed positive correlation of rigidity with balanced flexibility and family communication. Therefore, the short version of FACES IV (SAD_FACES) allows for the measurement of family functioning, especially emotional closeness and leadership and rules, relying on a limited number of items (24 items versus 42 items). This can bring several advantages to researchers willing to carry out research studies with samples of adolescents.
First, the agile structure of SAD_FACES can allow researchers to develop instruments which can include different scales avoiding 'side effects', such as the lack of the adolescents' attention when compiling long questionnaires, thereby putting less efforts in providing appropriate responses or skipping answers. Second, SAD_FACES can be a useful complement to investigations assessing the association of family functioning with other variables concerned with adolescents' wellbeing (e.g., Berge et al. 2013; Shek 1997; Suldo and Huebner 2004). Third, the short version of FACES IV allows for a more agile assessment of adolescents' individual wellbeing in relation to contextual dimensions that define adaptive or maladaptive family functioning.

\section{Limitations and Future Research}

Despite the strengths highlighted above, this validation study has some limitations, starting from the focus on a sample of middle adolescents belonging to a specific sociocultural context such as Italy. Future research should look at the validation of FACES IV across different age cohorts of adolescents, for instance 11-13 years and 17-19 years, and belonging to different cultural contexts. This could shed light on the observed association among the dimensions of the circumplex model. Moreover, further evidence is needed on the usage of FACES with clinical samples of adolescents; the agility of SAD_FACES, as stressed above, could facilitate investigations with this cohort.

\section{Compliance with Ethical Standards}

Conflict of Interest The authors declare that they have no conflict of interest.

Publisher's note Springer Nature remains neutral with regard to jurisdictional claims in published maps and institutional affiliations.

Open Access This article is licensed under a Creative Commons Attribution 4.0 International License, which permits use, sharing, adaptation, distribution and reproduction in any medium or format, as long as you give appropriate credit to the original author(s) and the source, provide a link to the Creative Commons license, and indicate if changes were made. The images or other third party material in this article are included in the article's Creative Commons license, unless indicated otherwise in a credit line to the material. If material is not included in the article's Creative Commons license and your intended use is not permitted by statutory regulation or exceeds the permitted use, you will need to obtain permission directly from the copyright holder. To view a copy of this license, visit http://creativecommons. org/licenses/by/4.0/.

\section{References}

Asparouhov, T., \& Muthén, B. (2009). Exploratory structural equation modeling: structural equation modeling. A Multidisciplinary Journal, 16(3), 397-438. 
Baiocco, R., Cacioppo, M., Laghi, F., \& Tafà (2013). Factorial and construct validity of FACES IV among Italian adolescents. Journal of Child and Family Studies, 22, 962-970.

Barnes, H., \& Olson, D. (1985). Parent adolescent communication and the circumplex model. Child Development, 56, 438-447.

Berge, J. M., Wall, M., Larson, N., Loth, K. A., \& Neumark-Sztainer, D. (2013). Family functioning: associations with weight status, eating behaviors, and physical activity in adolescents. Journal of Adolescent Health, 52, 351-357.

Comrey A.L., \& Lee, H.B. (2013). A first course in factor analysis. 2nd ed. NJ, Hillsdale: Lawrence Erlbaum Ass.

Everri, M., Fruggeri, L., \& Molinari, L. (2014). Microtransitions and the dynamics of family functioning. Integrative Psychological and Behavioral Science, 48, 61-78.

Everri, M., Mancini, T., \& Fruggeri, L. (2015). Family functioning, parental monitoring and adolescent familiar responsibility in middle and late adolescence. Journal of Child and Family Studies, 24, 3058-3066. https://doi.org/10.1007/s10826-0140109-z.

Everri, M., Mancini, T., \& Fruggeri, L. (2016a). Disentangling parental monitoring: the role of family communication in achieving parental knowledge. Psicologia Sociale, 3, 245-261.

Everri, M., Mancini, T., \& Fruggeri, L. (2016b). The role of Rigidity in adaptive and maladaptive families assessed by FACES IV: the points of view of adolescents. Journal of Child and Family Studies, 25, 2987-2997. https://doi.org/10.1007/s10826-016-0460-3.

Gosling, S. D., Rentfrow, P. J., \& Swann, Jr., W. B. (2003). A very brief measure of the big five personality domains. Journal of Research in Personality, 37, 504-528.

Graça Pereira, M., \& Teixeira, R. J. (2013). Portuguese validation of FACES-IV in adult children caregivers facing parental cancer. Contemporary Family Therapy, 35, 478-490.

Granger, C. V. (2007). Rasch analysis is important to understand and use for measurement. Rasch Measurement Transactions, 21(3), $1122-1123$.

Grimm, K. J., \& Widaman, K. F. (2012). Construct validity. In H. Cooper, P. M. Camic, D. L. Long, A. T. Panter, D. Rindskopf, \& K. J. Sher (Eds), APA handbooks in psychology ${ }^{\circledast}$. APA handbook of research methods in psychology, Vol. 1. Foundations, planning, measures, and psychometrics (pp. 621-642), American Psychological Association. https://doi.org/10.1037/13619-033.

Henry, C. S., Robinson, L. C., Neal, R. A., \& Erron, E. L. (2008). Adolescent perceptions of overall family system functioning and parental behaviors. Journal of Child and Family Studies, 15, 308-318.

Larson, R., Richards, M., Moneta, G., Holmbeck, G., \& Duckett, E. (1996). Changes in adolescents' daily interactions with their families from ages 10 to 18: disengagement and transformation. Developmental Psychology, 32, 744-754.

Loriedo, C., Di Nuovo, S., \& Visani, E. (2013). FACES IV: reliability and validity in an adult Italian sample. Minneapolis, MN: Life Innovations. http://www.facesiv.com.

Marsh, H. W. (2007). Application of confirmatory factor analysis and structural equation modeling in sport/exercise psychology. In G. Tenenbaum \& R. C. Eklund (Eds), Handbook of Sport Psychology (pp. 774-798). New York, NY: Wiley.

Marsh, H. W., Morin, A. J., Parker, P. D., \& Kaur, G. (2014). Exploratory structural equation modeling: an integration of the best features of exploratory and confirmatory factor analysis. Annual Review of Clinical Pychology, 10, 85-110.
Minuchin, S. (1974). Families and family therapy. Boston, MA: Harvard University Press.

Muthén, L. K. \& Muthén, B. O. (1998-2017). Mplus User's Guide. 8th ed. Los Angeles, CA: Muthén \& Muthén.

Myers, N. D., Ahn, S., \& Jin, J. (2011). Sample size and power estimates for a confirmatory factor analytic model in exercise and sport: a Monte Carlo approach. Research Quarterly for Exercise and Sport, 82, 412-423.

Olson, D. H. (2011). FACES IV and the circumplex model: validation study. Journal of Marital and Family Therapy, 37, 64-80.

Olson, D. H., \& Gorall, D. M. (2003). Circumplex model of marital and family systems. In F. Walsh (Ed), Normal family processes. 3rd ed. (pp. 514-548). New York: Guilford.

Olson, D. H., \& Gorall, D. M. (2006). Faces IV and the circumplex model. Minneapolis, MN: Life Innovations.

Olson, D. H., McCubbing, H. I., Barnes, H., Larsen, A., Muxen, M., \& Wilson, M. (1985). Family inventories: inventories in a national survey of families across the family life cycle. St. Paul: University of Minnesota, Family Social Science.

Olson, D. H., Russell, C. S., \& Sprenkle, D. H. (1989). Circumplex model: systematic assessment and treatment of families. New York, NY: Haworth Press.

Olson, D. H., Sprenkle, D. H., \& Russell, C. (1979). Circumplex model of marital and family systems: I. Cohesion and adaptability dimensions, family types, and clinical applications. Family Process, 18, 3-28.

Perry, J. L., Nicholls, A. R., Clough, P. J., \& Crust, L. (2015). Assessing model fit: caveats and recommendations for confirmatory factor analysis and exploratory structural equation modeling. Measurement in Physical Education and Exercise Science, 19, 12-21. https://doi.org/10.1080/1091367X.2014.952370.

Postmes, T., Haslam, S. A., \& Jans, L. (2013). A single-item measure of social identification: reliability, validity, and utility. British Journal of Social Psychology, 52, 597-617. https://doi.org/10. 1111/bjso.12006.

R Core Team. (2019). R: a language and environment for statistical computing. Vienna, Austria: R Foundation for Statistical Computing. http://www.r-project.org/index.html.

Rivero, N., Martinez-Pampliega, A., \& Olson, D. (2010). Spanish adaptation of the FACES IV Questionnaire: psychometric characteristics. The Family Journal, 18, 288-296.

Shek, D. T. L. (1997). The relation of family functioning to adolescent psychological well-being, school adjustment, and problem behavior. Journal of Genetic Psychology, 158, 467-479.

Suldo, S. M., \& Huebner, E. S. (2004). The role of life satisfaction in the relationship between authoritative parenting dimensions and adolescent problem behavior. Social Indicators Research, 66, 165-195.

Tóth-Király, I., Bõthe, B., Rigó, A., \& Orosz, O. (2017). An illustration of the exploratory structural equation modeling (ESEM) framework on the passion scale. Frontiers in Psychology, 1968, $1-15$.

van Buuren, S., \& Groothuis-Oudshoorn, K. (2011). Mice: multivariate imputation by chained equations in R. Journal of Statistical Software, 3, 1-67.

Ziegler, M., Kemper, C. J., \& Kruyen, P. (2014). Short scales-five misunderstandings and ways to overcome them. Journal of Individual Differences, 35(4), 185-189. https://doi.org/10.1027/ 1614-0001/a000148. 